\title{
No Clear, Direct Evidence for Multiple Protoplanets Orbiting LkCa 15: LkCa 15 bed are Likely Inner Disk Signals
}

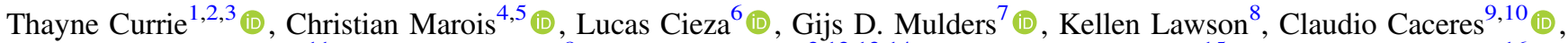
Dary Rodriguez-Ruiz $^{11}$, John Wisniewski ${ }^{8}$ (D) Olivier Guyon ${ }^{2,12,13,14}$ (D), Timothy D. Brandt ${ }^{15}$ (D), N. Jeremy Kasdin ${ }^{16}$, Tyler D. Groff ${ }^{17}$, Julien Lozi ${ }^{2}$, Jeffrey Chilcote ${ }^{18}$ (D), Klaus Hodapp ${ }^{19}$ (D), Nemanja Jovanovic ${ }^{20}$, Frantz Martinache ${ }^{21}$ (D), Nour Skaf ${ }^{2,22}$, Wladimir Lyra ${ }^{23,24}$ (D) , Motohide Tamura ${ }^{14,25,26}$ (i), Ruben Asensio-Torres ${ }^{27}$ (i), Ruobing Dong ${ }^{5}$ (iD), Carol Grady ${ }^{3,17}$, Benjamin Gerard $^{28}$, Misato Fukagawa ${ }^{26}$ (D), Derek Hand ${ }^{2}$, Masahiko Hayashi ${ }^{26}$, Thomas Henning ${ }^{29}$, Tomoyuki Kudo ${ }^{2}$ (D), Masayuki Kuzuhara $^{14}$, Jungmi Kwon ${ }^{30}$ (D), Michael W. McElwain ${ }^{17}$, and Taichi Uyama ${ }^{25}$ (D)

${ }^{1}$ NASA-Ames Research Center, Moffett Boulevard, Moffett Field, CA, USA; thayne.m.currie@nasa.gov, currie@naoj.org

${ }^{2}$ Subaru Telescope, National Astronomical Observatory of Japan, 650 North A'ohōkū Place, Hilo, HI 96720, USA

${ }^{3}$ Eureka Scientific, 2452 Delmer Street, Suite 100, Oakland, CA, USA

${ }^{4}$ National Research Council of Canada Herzberg, 5071 West Saanich Road, Victoria, BC, V9E 2E7, Canada

${ }^{5}$ University of Victoria, 3800 Finnerty Road, Victoria, BC, V8P 5C2, Canada

${ }^{6}$ Núcleo de Astronomía, Facultad de Ingeniería y Ciencias, Universidad Diego Portales, Av Ejército 441, Santiago, Chile

${ }_{8}^{7}$ Department of the Geophysical Sciences, The University of Chicago, Chicago, IL 60637, USA

${ }^{8}$ Homer L. Dodge Department of Physics, University of Oklahoma, Norman, OK 73071, USA

${ }^{9}$ Departamento de Ciencias Fisicas, Facultad de Ciencias Exactas, Universidad Andres Bello. Av. Fernandez Concha 700, Las Condes, Santiago, Chile

${ }^{10}$ Núcleo Milenio Formación Planetaria-NPF, Universidad de Valparaíso, Av. Gran Bretaña 1111, Valparaíso, Chile

${ }^{11}$ Department of Physics and Astronomy, Rochester Institute of Technology, Rochester, NY, USA

${ }^{12}$ Steward Observatory, University of Arizona, Tucson, AZ 85721, USA

${ }_{13}$ College of Optical Sciences, University of Arizona, Tucson, AZ 85721, USA

${ }^{14}$ Astrobiology Center of NINS, 2-21-1, Osawa, Mitaka, Tokyo, 181-8588, Japan

${ }_{15}$ Department of Physics, University of California, Santa Barbara, Santa Barbara, CA, USA

${ }^{16}$ Department of Mechanical Engineering, Princeton University, Princeton, NJ, USA

${ }^{17}$ NASA-Goddard Space Flight Center, Greenbelt, MD, USA

${ }^{18}$ Department of Physics, University of Notre Dame, South Bend, IN, USA

${ }^{19}$ Institute for Astronomy, University of Hawaii, 640 North A'ohōkū Place, Hilo, HI 96720, USA

${ }^{20}$ Department of Astronomy, California Institute of Technology, 1200 East California Boulevard, Pasadena, CA 91125, USA

${ }^{21}$ Université Côte d'Azur, Observatoire de la Côte d'Azur, CNRS, Laboratoire Lagrange, France ${ }^{22}$ Imperial College London, Kensington, London SW7 2AZ, UK

${ }^{23}$ Department of Physics and Astronomy, California State University Northridge, 18111 Nordhoff Street, Northridge, CA 91130, USA

${ }^{24}$ Jet Propulsion Laboratory, California Institute of Technology, 4800 Oak Grove Drive, Pasadena, CA 91109, USA

${ }^{25}$ Department of Astronomy, Graduate School of Science, The University of Tokyo, 7-3-1, Hongo, Bunkyo-ku, Tokyo, 113-0033, Japan

${ }^{26}$ National Astronomical Observatory of Japan, 2-21-2, Osawa, Mitaka, Tokyo 181-8588, Japan

${ }^{27}$ Department of Astronomy, Stockholm University, AlbaNova University Center, SE-106 91 Stockholm, Sweden

${ }^{28}$ University of Victoria, 3800 Finnerty Rd, Victoria, BC, V8P 5C2, Canada

${ }^{29}$ Max Planck Institut fur Astronomie, Konigstuhl 17, D-69117 Heidelberg, Germany

${ }^{30}$ ISAS/JAXA, 3-1-1 Yoshinodai, Chuo-ku, Sagamihara, Kanagawa 252-5210, Japan

Received 2019 February 20; revised 2019 April 18; accepted 2019 April 21; published 2019 May 15

\begin{abstract}
Two studies utilizing sparse aperture-masking (SAM) interferometry and $\mathrm{H}_{\alpha}$ differential imaging have reported multiple Jovian companions around the young solar-mass star, LkCa 15 (LkCa 15 bcd): the first claimed direct detection of infant, newly formed planets ("protoplanets"). We present new near-infrared direct imaging/spectroscopy from the Subaru Coronagraphic Extreme Adaptive Optics (SCExAO) system coupled with Coronagraphic High Angular Resolution Imaging Spectrograph (CHARIS) integral field spectrograph and multi-epoch thermal infrared imaging from Keck/ NIRC2 of LkCa 15 at high Strehl ratios. These data provide the first direct imaging look at the same wavelengths and in the same locations where previous studies identified the LkCa 15 protoplanets, and thus offer the first decisive test of their existence. The data do not reveal these planets. Instead, we resolve extended emission tracing a dust disk with a brightness and location comparable to that claimed for LkCa 15 bcd. Forward-models attributing this signal to orbiting planets are inconsistent with the combined SCExAO/CHARIS and Keck/NIRC2 data. An inner disk provides a more compelling explanation for the SAM detections and perhaps also the claimed $\mathrm{H}_{\alpha}$ detection of $\mathrm{LkCa} 15 \mathrm{~b}$. We conclude that there is currently no clear, direct evidence for multiple protoplanets orbiting $\mathrm{LkCa} 15$, although the system likely contains at least one unseen Jovian companion. To identify Jovian companions around $\mathrm{LkCa} 15$ from future observations, the inner disk should be detected and its effect modeled, removed, and shown to be distinguishable from planets. Protoplanet candidates identified from similar systems should likewise be clearly distinguished from disk emission through modeling.
\end{abstract}

Key words: instrumentation: high angular resolution - planetary systems - stars: individual (LkCa 15) - stars: variables: $\mathrm{T}$ Tauri, Herbig $\mathrm{Ae} / \mathrm{Be}$ - techniques: image processing

\section{Introduction}

Young, 1-10 Myr old Jovian protoplanets embedded in disks around newly born stars provide a crucial link between the first stages of planet formation and the properties of directly imaged, fully formed planets orbiting 10-100 Myr old stars (e.g., Marois et al. 2008b, 2010b). LkCa 15, a solar-mass T Tauri star and 
member of the 1-3 Myr old Taurus-Auriga star-forming region (Kenyon et al. 2008), is a superb laboratory for studying planet formation and searching for protoplanets. The star is surrounded by an accreting, gas-rich protoplanetary disk with multiple dust components: hot $\left(T_{\text {eff }}=1400 \mathrm{~K}\right)$, sub-au scale dust producing broadband near-infrared (NIR) excess and cooler massive outer dust, which are separated by a solar system-scale cavity plausibly created by Jovian protoplanets (Espaillat et al. 2007; Thalmann et al. 2010; Andrews et al. 2011; Dodson-Robinson \& Salyk 2011; Dong \& Fung 2017; Alencar et al. 2018).

Using sparse aperture masking interferometry (SAM; Tuthill et al. 2006) of LkCa 15, Kraus \& Ireland (2012) reported the detection of one protoplanet located within an ostensibly cleared gap in dust emission. Also using SAM, Sallum et al. (2015b) then identified three protoplanets within $\rho \sim 0$ !" $15(\approx 25 \mathrm{au})$ (LkCa 15 bcd), one of which was recovered in $\mathrm{H}_{\alpha}$ (LkCa $15 \mathrm{~b}$ ). Thus, LkCa 15 appeared to show evidence for multiple Jovian protoplanets, the first such system ever reported.

However, the closure phase signals of disks in SAM data can mimic those of protoplanets (Cieza et al. 2013; Kraus et al. 2013). LkCa 15's circumstellar environment as seen in scattered light is complex, including a bright outer dust wall (Thalmann et al. 2010, 2014). Additionally, inner dust disk material is now resolved at optical wavelengths and NIR polarimetry out to $\mathrm{LkCa} 15$ bcd-like separations (Oh et al. 2016b; Thalmann et al. 2016). Depending on this dust disk's brightness and spatial extent in (a) total intensity at (b) the longer wavelengths where $\mathrm{LkCa} 15 \mathrm{bcd}$ were identified $(2.2-3.8 \mu \mathrm{m})$, it could instead be the signal masquerading as these protoplanets. However, previous $2.2-3.8 \mu \mathrm{m}$ total intensity data lack the image quality/sensitivity to probe these regions (Thalmann et al. 2014).

In this Letter, we use multi-epoch direct imaging observations of LkCa 15 obtained from the Subaru Coronagraphic Extreme Adaptive Optics (SCExAO) project coupled with the Coronagraphic High Angular Resolution Imaging Spectrograph (CHARIS) in the NIR $(J H K / 1.1-2.4 \mu \mathrm{m}$; Groff et al. 2015, 2017; Jovanovic et al. 2015) and Keck/NIRC2 in the thermal infrared $\left(L_{\mathrm{p}} / 3.78 \mu \mathrm{m}\right)$. These data provide the first direct imaging look at the same wavelengths and in the same locations where previous studies identified the LkCa 15 protoplanets $(K$, $L_{\mathrm{p}}$ ), and thus offer the first decisive test of their existence.

\section{Observations and Data Reduction}

\subsection{SCEXAO/CHARIS JHK Direct Imaging/Spectroscopy}

We observed LkCa 15 on UT 2017 September 07 and UT 2018 January 8 using SCExAO coupled with CHARIS operating in low-resolution $(R \sim 20)$, broadband mode, covering the $J H K$ filters simultaneously ( $t_{\text {int }}=31$ and 19 minutes). All data were acquired in angular differential imaging mode (ADI; Marois et al. 2006). For the September data, given our modest parallactic angle rotation $\left(\triangle \mathrm{PA}=60^{\circ}\right)$, we also observed a nearby, near-color matched star (V819 Tau) as a contemporaneous point-spread function (PSF) reference. ${ }^{31}$ Conditions were excellent (0".3-0."4 $V$-band seeing). Despite LkCa 15's and V819 Tau's optical faintness $(R \sim 11.6,12.2)$, we achieved

\footnotetext{
31 V819 Tau has a marginal unresolved infrared (IR) excess longward of 10-15 $\mu \mathrm{m}$ (Furlan et al. 2009). However, we find no hint of a disk in SCExAO/CHARIS data or in a separate Keck/NIRC2 $L_{\mathrm{p}}$ data set. Subaru/ HiCIAO $H$-band polarimetry data show that V819 Tau is a non-detection for any disk (J. Hashimoto 2019, private communication). For our purposes, V819 Tau is effectively a bare stellar photosphere.
}

high-quality corrections with a diffraction-limited PSF and the first 8-9 Airy rings visible. While we could not directly estimate the Strehl ratio, raw contrasts were similar to those for other stars for which SCExAO's real-time telemetry monitor reported $\approx 70 \%$ Strehl in $H$ band. For the January data, conditions were poorer and we did not observe a PSF reference star, but the parallactic angle motion was larger $\left(120^{\circ}\right)$.

Spectral extraction utilized the cube rectification pipeline from Brandt et al. (2017) and basic image processing was performed as in Currie et al. (2018a, 2018b). A model spectral energy distribution (SED) reproducing $\mathrm{LkCa} 15$ 's broadband photometry provided spectrophotometric calibration. ${ }^{32}$ No coronagraphs or satellite spots were used; all stellar PSFs were unsaturated.

\subsection{Keck/NIRC2 $L_{\mathrm{p}}$ Direct Imaging}

First, we reduced multiple LkCa 15 high-contrast imaging data sets from the Keck Observatory Archive with more than $2 \lambda / D$ parallactic angle rotation at $\mathrm{LkCa} 15$ bcd's reported angular separation, selecting 2009 November $21 L_{\mathrm{p}}$ data (PI: L. Hillenbrand; $\Delta \mathrm{PA}=132^{\circ} .5, t_{\text {int }}=5.4$ minutes). These data have the highest quality of those taken without a coronagraph that may partially occult $\mathrm{LkCa} 15$ bcd and are contemporaneous with the first aperture-masking detection reported in Kraus \& Ireland (2012). Second, we obtained NIRC2 data on 2017 December 9 and 10 for 17.6 and 13.8 minutes with $150^{\circ}$ and $160^{\circ}$ parallactic motion. LkCa 15 was observed continuously through transit on the first night; on the second night, we alternated between it and a diskless PSF reference star (V1075 Tau). All data were acquired in ADI mode using the narrow camera with various dither patterns.

Keck/NIRC2's adaptive optics (AO) system delivered median Strehl ratios of 0.79 and $0.77-0.79$ in $L_{\mathrm{p}}$ for the 2009 November and two 2017 December data sets, as measured by a modified (for the appropriate pixel scale) observatory-supplied routine nirc2strehl.pro. Stars were unsaturated in all images. Basic processing followed previous steps used for thermal-IR data with our well-tested broadband imaging pipeline (Currie et al. 2011, 2014b), including a linearity correction, sky subtraction, distortion correction and bad pixel interpolation, image registration, and flux normalization.

\subsection{PSF Subtraction}

Because of the complex astrophysical scene within $\rho \sim 0$ ". 5 of $\mathrm{LkCa} 15$, extreme care is needed to properly perform PSF subtraction to avoid misinterpretation (Currie et al. 2017a). For systems like LkCa 15 observed in ADI mode, bright, spatially varying protoplanetary disk emission changes in position angle on the detector over the course of an observing sequence, "corrupting" the covariance matrices utilized in powerful, widely used least-squares approaches like the Locally Optimized Combination of Images (LOCI) and Karhunen-Loéve Image Projection (KLIP) algorithms plus successors (Lafreniére et al. 2007; Marois et al. 2010a, 2014; Currie et al. 2012; Soummer et al. 2012). Additionally, at very small angles, morphological biasing of an astrophysical source in ADI due to self-subtraction can be severe.

\footnotetext{
32 LkCa 15 exhibits small-amplitude variability at optical and mid-IR (MIR) wavelengths (Espaillat et al. 2011; Rodriguez et al. 2017), with a peak-to-peak value of $\sim 0.1 \mathrm{mag}$. No clear evidence establishes that $\mathrm{LkCa} 15$ is variable at qualitatively greater level in the $J H K$ bands, let alone at a level that could affect our conclusions.
} 

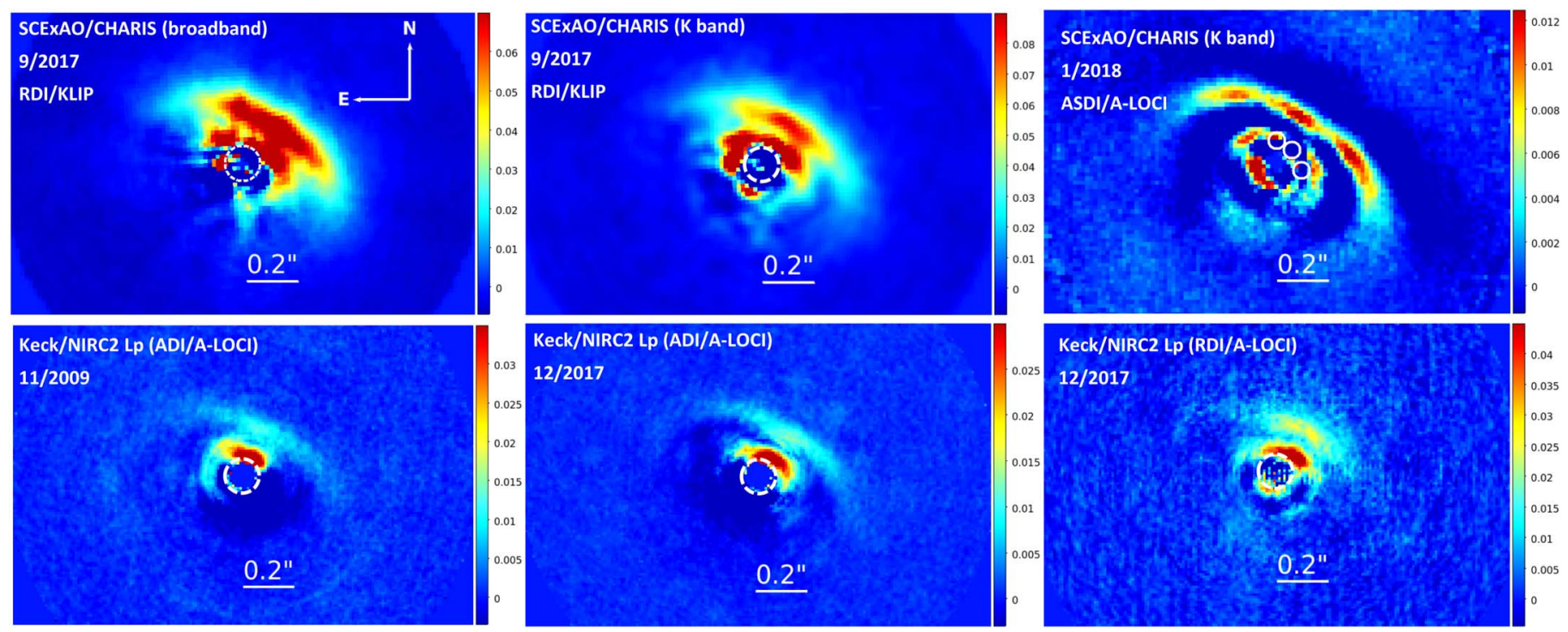

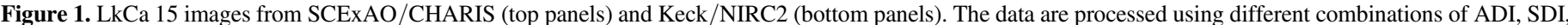

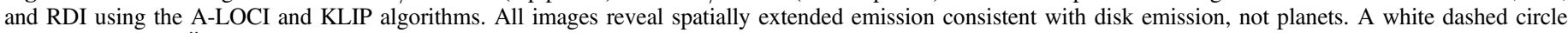

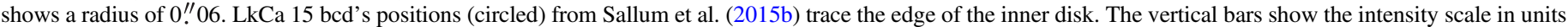
of mJy normalized to one FWHM.

Therefore, we adopted the following approach. First, for data sets obtained with a suitable PSF reference star, we performed reference star differential imaging (RDI) using KLIP and Adaptive Locally Optimized Combination of Images (A-LOCI; Currie et al. 2012), where we equate the region used to construct a weighted reference PSF (the optimization zone) and the region over which this PSF is subtracted (the subtraction zone) with the outer radius set to the visible PSF halo, beyond the angles covered by LkCa 15 's disk structures ( $\rho \approx 0$ "'75-1". 1 ). Second, we performed an ADI-based reduction using A-LOCI on the other data sets using an optimization zone also extending to the PSF halo edge, constructing a weighted reference PSF used to attenuate speckles over smaller annular subtraction zones ( $\Delta r=2.5-5$ pixels). To further reduce algorithm "aggressiveness," we applied a rotation gap of $\delta \approx 0.5-1 \quad \lambda / D$, while truncating the covariance matrix's diagonal terms with singular value decomposition. To better suppress residual speckles with the 2018 January CHARIS data, we performed a classical SDI reduction (median-combination of channels re-scaled by wavelength) on the ADI/A-LOCI residuals. ${ }^{33}$

\section{Detection of the LkCa 15 Inner Dust Disk and Non-detection of LkCa 15 bcd}

Figure 1 shows the SCExAO/CHARIS NIR images in broadband (a median-combination of all channels) and in $K$ band (top panels) and Keck/NIRC2 $L_{\mathrm{p}}$ images (bottom panels). All data easily resolve the forward-scattering side of the crescent-shaped outer dust disk wall (e.g., Thalmann et al. 2010, 2014). However, no data set reveals direct evidence for LkCa 15 bcd. Instead, all data resolve another crescent-shaped extended structure interior to the outer disk wall, consistent with the wall of an inner dust disk previously only seen in polarized light (Thalmann et al. 2015; Oh et al. 2016b).

Inspection of individual CHARIS data cubes and NIRC2 images shows that this extended inner disk emission cannot be

\footnotetext{
33 CHARIS's large bandpass enables SDI while only partially annealing point sources at $\mathrm{LkCa} 15$ bcd-like separations.
}

explained by residual speckle noise that is preserved when images are derotated and combined (for CHARIS and NIRC2) or wavelength-collapsed (for CHARIS). RDI-reduced images obtained using a range of principal components (for KLIP) or a range of SVD cutoffs (for A-LOCI) all recover the same structure. For CHARIS, the inner disk is visible in most individual channels, especially those covering the $H$ and $K$ passbands. Furthermore, ADI and ASDI-reduced images (2018 January CHARIS data and two of the three Keck/NIRC2 data sets) also show negative selfsubtraction footprints of this inner disk. ${ }^{34}$

We further confirmed that we could have detected LkCa 15 bcd-like planets in absence of disk emission. To empirically assess our sensitivity to point sources, we injected and attempted to recover model planets with an early L dwarflike spectrum into our raw $\mathrm{LkCa} 15$ data reduced with RDI (2017 September CHARIS data and 2017 December NIRC2 data). We considered the half-field of view opposite the peak brightness of the inner disk and at a range of angular separations. ${ }^{35}$ We varied the brightnesses of these planets with respect to the star to be equal to or fainter than that for $\mathrm{LkCa} 15$

\footnotetext{
${ }^{34}$ A separate ASDI reduction of the 2017 September CHARIS data and reduction of other data sets not considered here-an ADI reduction of archival 2016 October $K_{\mathrm{s}}$ SCExAO/HiCIAO data, and ADI reductions of additional archival Keck/NIRC2 $M_{\mathrm{p}}$ and $L_{\mathrm{p}}$ data from 2012 and 2015-likewise show a detection of the inner disk, not planets, albeit with more residual speckle contamination and/or poorer sensitivity.

35 Typically, contrast curves are derived numerically based on the radial noise profile (e.g., Marois et al. 2008a; Currie et al. 2011). However, at small angles relevant for this study, corrections to the nominal 3-5 $\sigma$ limits due to finite sample sizes (Mawet et al. 2014) are significant. In particular, the contrast penalty to achieve a Gaussian noise-equivalent $5 \sigma$ limit at $1-2 \lambda / D$ with a False Positive Fraction (FPF) of $\sim 2.86 \times 10^{-7}$ is prohibitively large for a halffield of view (see Figure 6 in Mawet et al. 2014). Setting the FPF to $1.35 \times 10^{-3}$ as recommended by Mawet et al. (2014) for the smallest angles, equivalent to the FPF for a $3 \sigma$ detection in Gaussian statistics, shows that planets with brightnesses comparable $\mathrm{LkCa} 15$ bcd would in fact be recovered at the $>3 \sigma$ level despite residual disk emission. While residual disk emission at small angles causes the true noise to be overestimated, a substantial positive skew in the noise profile (which itself is uncertain due to finite sample sizes) can cause the FPF to be underestimated (Marois et al. 2008a; Currie et al 2014a). For all these complications, we opt for a more direct, empirical approach of injecting and recovering planets with known contrasts.
} 

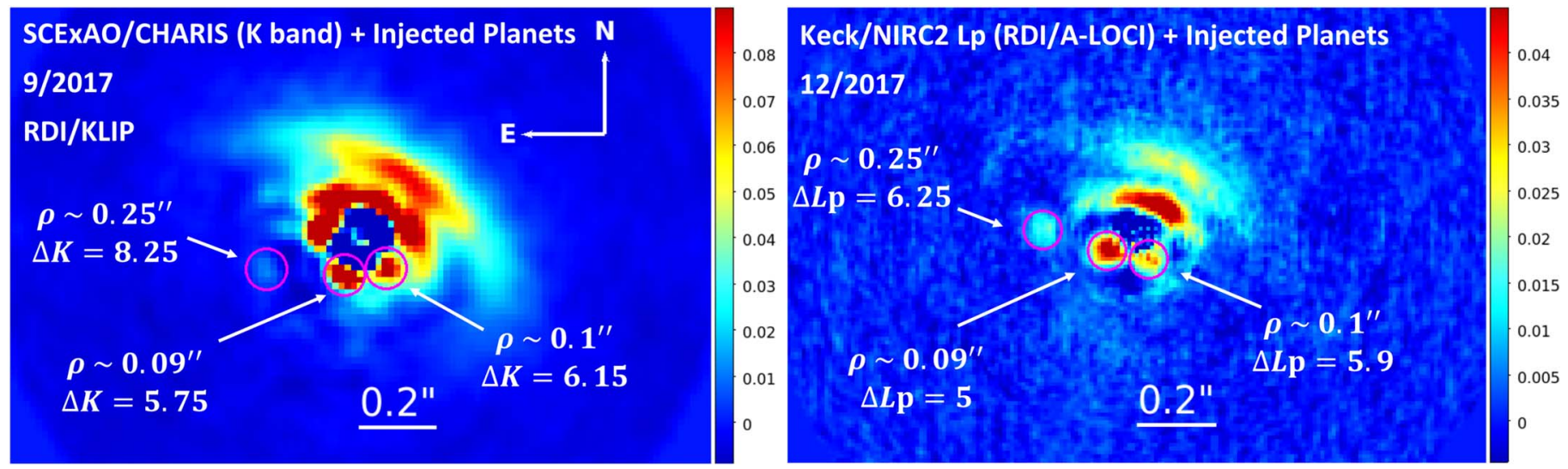

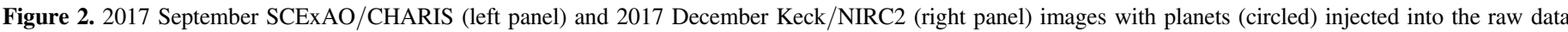

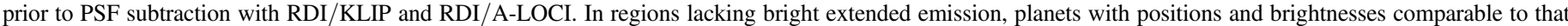

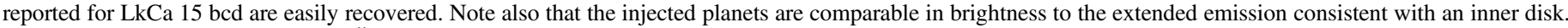
At slightly wider separations $(\rho \sim 0$ ". 25 ), planets over $1.25-2.5$ mag fainter than LkCa 15 bcd are detectable.

bc at $K$ and $L_{\mathrm{p}}$ as reported by Sallum et al. (2015b): $\Delta K \sim$ 5.5-6 and $\Delta L_{\mathrm{p}} \sim 5-5.9$.

Figure 2 shows example RDI-reduced SCExAO/CHARIS and Keck/NIRC2 data sets with injected planets. The planets' throughputs are high, ranging between $75 \%$ and $100 \%$. In spite of some contamination from residual inner disk emission, planets with separations comparable to $\mathrm{LkCa} 15$ bcd $(\rho \sim 0$ ". 09-0!' 1$)$ are detected at LkCa 15 bed-like contrasts $\left(\Delta K \sim 5.75,6.15 ; \Delta L_{\mathrm{p}} \sim 5,5.9\right)$ and visible as point sources. The contrasts of these recovered planets are similar to limits achieved for diskless stars with SAM in Kraus et al. (2011) and Lacour et al. (2011). Planets even fainter than proposed for LkCa 15 bcd- $\Delta K \sim 6.5, \Delta L_{\mathrm{p}} \sim 6.3$-are detected and identified as point sources at $\rho \sim 0$ ". $09-0$ ". 1 in regions of the lowest disk emission (not shown). At wider separations ( $\rho \gtrsim 0$ "! 4 , our contrast limits are equal to or deeper than achievable with SAM $\left(\Delta K, \Delta L_{\mathrm{p}} \sim 10,7.5\right) .{ }^{36}$

Comparisons between our images and SAM results strongly suggest that this inner disk emission is the same astrophysical source previously interpreted as the $\mathrm{LkCa} 15$ bcd protoplanets. For both CHARIS and NIRC2 data, the inner disk emission extends from $\rho \sim 0$ ". 07 to $\rho \sim 0$ "' 25 ( $r_{\text {proj }} \approx 10-40$ au) with an apparent semimajor and semiminor axis for the emission's peak at $\rho \sim 0$ "! 2 and 0 ." 1 , respectively $\left(r_{\text {proj }} \approx 17-32 \mathrm{au}\right)$. In the RDI-reduced data sets, the emission subtends an angle of $\sim 100^{\circ}$, which is roughly the same position angle range for LkCa 15 bcd reported in Sallum et al. (2015b). Planet positions reported in Sallum et al. (2015b; circles in the 2018 January CHARIS data) trace this emission. The aggregate flux density for LkCa 15 bcd from Sallum et al. (2015b) is $\approx 3.7 \pm 1.2 \mathrm{mJy}$ and $5.4 \pm 1.5$ in $K$ and $L_{\mathrm{p}}$, respectively. Over the same range of position angles/separations reported for $\mathrm{LkCa}$ 15 bcd, the summed inner disk flux densities in the CHARIS $K$ band and NIRC2 $L_{\mathrm{p}}$ data reduced using RDI are the same, within uncertainties $^{37}: \approx 2.8$ and $3.9 \mathrm{mJy}$.

\footnotetext{
36 For the 2009 NIRC2 $L_{\mathrm{p}}$ data reduced with ADI/A-LOCI, the forwardscattering peak of the inner disk severely self-subtracts point sources injected into the data at $\rho \sim 0$ " 1 : thus, injecting planets into these data as performed in for our RDI-reduced data sets substantially underestimates our true sensitivity in absence of a disk. Nevertheless, planets with LkCa 15 bc-like contrasts are still detectable at LkCa 15 bcd-like separations as well.

37 As we found in the immediate preceding analysis, RDI processing induces only modest signal loss for point sources and disks at $\mathrm{LkCa} 15$ bcd-like separations: the throughput-corrected flux density for the inner disk still matches that reported for $\mathrm{LkCa} 15$ bcd combined together.
}

LkCa 15 images obtained at different wavelengths reveal some evidence for color differences between the spatially resolved inner and outer disk components. In the SCExAO/ CHARIS broadband image $\left(J H K, \lambda_{\mathrm{o}}=1.63 \mu \mathrm{m}\right)$, the peak brightness of the inner component is about $30 \%$ higher than the peak of the outer component. At $K$-band $\left(\lambda_{\mathrm{o}}=2.18 \mu \mathrm{m}\right)$, the peak brightness of the inner disk is about 1.75 times than the outer disk, while at $L_{\mathrm{p}}$ the inner disk is more than twice as bright as the outer disk. The physical origin of these differences will be addressed in Section 4.2.

\section{Forward-modeling of LkCa 15 Images: A Forward- scattering Inner Dust Disk, Not Multiple Orbiting Planets}

We now compare the LkCa 15 images to forward-models (Marois et al. 2010a) for LkCa 15 bcd and an inner disk. Our analysis adopts the approaches in Pueyo (2016) and Currie et al. (2018b) for KLIP and A-LOCI, using the eigenvalues/eigenvectors in KLIP or coefficients in A-LOCI drawn from the real data and applying them to synthetic planet/disk signals injected into empty data cubes/images. Our goal is to (1) confirm that the emission we interpret as an inner disk cannot be reproduced by properties previously attributed to $\mathrm{LkCa} 15 \mathrm{bcd}$, and (2) then explore the general properties of this inner disk.

We focused on the highest-quality data easily amenable to forward-modeling at wavelengths where $\mathrm{LkCa} 15 \mathrm{bcd}$ were identified $\left(K, L_{\mathrm{p}}\right)$. Thus, we considered the $K$-band portion of the 2017 September SCExAO/CHARIS data processed with RDI/KLIP, the 2009 November NIRC2 $L_{\mathrm{p}}$ data processed with ADI/A-LOCI, and the 2017 December NIRC2 $L_{\mathrm{p}}$ data processed with RDI/A-LOCI.

\subsection{Planet Forward-modeling}

We produced forward-models of (a) all three planets ( $\mathrm{LkCa}$ $15 \mathrm{bcd}$ ) and (b) just the two identified in Sallum et al. (2015b) from multiple epochs ( $\mathrm{LkCa} 15 \mathrm{bc}$ ), (1) at the planets' last reported positions in Sallum et al. (2015b) in 2014 November2015 February, and (2) at the planets' estimated positions in 2009 November, 2017 September, and 2017 December. To predict the planets' positions in multiple epochs, we adopted the Sallum et al. astrometry and the Gaia second data release (DR2) distance to $\mathrm{LkCa} 15(158.9 \mathrm{pc})$, assuming that the planets are on circular orbits in the same plane as the outer disk 

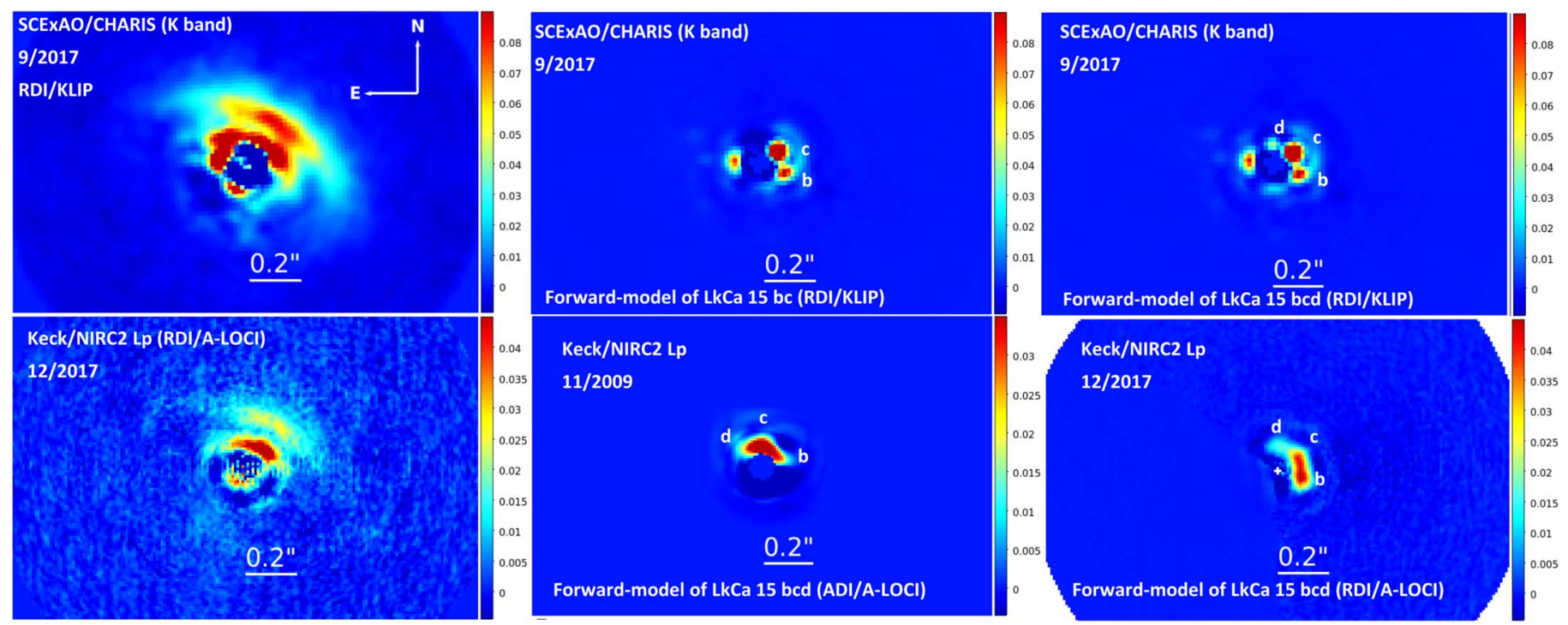

Figure 3. Comparisons between our observed data (left panels) and forward-models of LkCa 15bcd (middle/right panels) for the 2017 September SCExAO/CHARIS data (top) and 2009 November and 2017 December Keck/NIRC2 data (bottom). The predicted positions for LkCa 15 bcd in 2009 November and 2017 September/ December are $\rho \sim 00^{\prime \prime} 082, \mathrm{PA}=-74^{\circ}$ and $\rho \sim 00^{\prime \prime} 1, \mathrm{PA}=-109^{\circ}$ for LkCa $15 \mathrm{~b} ; \rho \sim 00^{\prime \prime} 083, \mathrm{PA}=-6^{\circ}$ and $\rho \sim 00^{\prime \prime} 085, \mathrm{PA}=-60^{\circ}$ for LkCa $15 \mathrm{c}$; and $\rho \sim 0{ }^{\prime \prime} 1, \mathrm{PA}=39^{\circ}$ and $\rho \sim 00^{\prime \prime} 08, \mathrm{PA}=-1^{\circ}$ for LkCa $15 \mathrm{~d}$.

$\left(i \sim 50^{\circ}, P A_{\text {minor }} \sim 60^{\circ}\right.$; Thalmann et al. 2014, 2015; Oh et al. 2016b). Their deprojected orbital separations in 2014 November-2015 February are $~ 16-18$ au; their position angles change by $\approx 5^{\circ} \mathrm{yr}^{-1}$ in the orbital plane.

We adopted the Sallum et al. (2015b) $L_{\mathrm{p}}$ photometry for LkCa 15 bcd. In $K$, we also adopted their LkCa 15 bc photometry. LkCa 15 d has no claimed detection in $K$ from Sallum et al. (2015b). We assumed that LkCa 15 d's $K-L_{\mathrm{p}}$ colors are similar to LkCa 15 bc's and thus adopted $\Delta K=7$.

Figure 3 shows forward-models of the $\mathrm{LkCa} 15$ planets for CHARIS $K$-band (top panels) and NIRC2 $L_{\mathrm{p}}$ (bottom panels). The emission's apparent brightness in the CHARIS data is comparable to the combined brightness proposed for $\mathrm{LkCa} 15$ bcd. However, LkCa 15 bcd would be clearly distinguishable as separate point sources in $K$, whereas the CHARIS data instead show a continuous structure. Thus, the SCExAO/CHARIS data are inconsistent with planets being responsible for this emission.

At $L_{\mathrm{p}}$, LkCa 15 bcd's PSFs are partially blended. ${ }^{38}$ However, due to orbital motion, the aggregate emission from $\mathrm{LkCa} 15$ bc(d) should rotate clockwise by $\sim 35^{\circ}-40^{\circ}$ between 2009 and 2017: the emission centroid, measured in the forward-modeled planet images from regions within $50 \%$ of the peak intensity, changes by $\approx 1 \lambda / D$. In contrast, the measured center of mass for this emission in the real 2009 and 2017 data is constant to within $0.05-0.1 \lambda / D$, implying a static morphology over $8 \mathrm{yr}$. Thus, the Keck/NIRC2 data are inconsistent with planetary orbital motion.

\subsection{Disk Forward-modeling}

To explore the general properties of inner disk emission previously attributed to $\mathrm{LkCa} 15 \mathrm{bcd}$, we produced and then forward-modeled synthetic scattered-light disk images with

\footnotetext{
38 On the other hand, a forward-model including only LkCa $15 \mathrm{bc}$, resembling the reconstructed images from 2009 November SAM data (Kraus \& Ireland 2012; Sallum et al. 2016), is morphologically inconsistent with our real 2009 November data, as it would reveal the planets as separate point sources. The SAM image reconstructions in some cases are therefore not faithfully reproducing the spatial distribution of astrophysical signals near LkCa 15 .
}

SCExAO/CHARIS using the MCMax3D radiative transfer code (Min et al. 2009), adopting the formalism from Mulders et al. (2010, 2013). Our approach considered three spatially extended components: (1) an optically thick (sub-)au scale hot component responsible for the NIR broadband excess and $10 \mu \mathrm{m}$ silicate feature, (2) a warm component responsible for the inner disk resolved with SCExAO/CHARIS and Keck/NIRC2, and (3) the optically thick outer disk, which has been resolved in optical/NIR scattered light (e.g., Thalmann et al. 2014, 2016) and with (sub-) millimeter data (Andrews et al. 2011; Isella et al. 2014). Following Thalmann et al. (2014, 2016), we envisioned that components 1 and 2 shadow and may be slightly misaligned with the outer disk (component 3). We explored a small range of component parameters, settling on a fiducial model with properties listed in Table 1. Except for a few Spitzer/IRS channels probing the unresolved sub-au component, the model fits $\mathrm{LkCa} 15$ 's entire SED from the optical to millimeter to within $~ 20 \%-30 \%$ (Andrews et al. 2011; Isella et al. 2014; Ribas et al. 2017).

Figure 4 compares the SCExAO/CHARIS broadband image (top) and Keck/NIRC2 $L_{\mathrm{p}}$ image with our fiducial model. The PSF-subtracted model reproduces the brightness and morphology of the inner/outer disk components: the subtraction residuals do not reveal any emission consistent with $\mathrm{LkCa} 15$ bcd. The peak pixel intensity at positions covering LkCa $15 \mathrm{bcd}$ (circled) is always less than 1/3 (1/4) that predicted for LkCa 15 b(c). Residuals at $\rho \lesssim 0$ !! 2 that do remain are attributable to slight mismatches with extended disk emission. ${ }^{39}$

While a wide range of models match either the SCExAO/ CHARIS or Keck/NIRC2 data, the combined data point toward different grain properties for the three disk components. A larger minimum dust grain size for the resolved inner disk versus resolved outer disk $(\sim 0.6 \mu \mathrm{m}$ versus $0.1 \mu \mathrm{m})$ better reproduces the inner disk's redder color and more pronounced

\footnotetext{
${ }^{39}$ For example, weak residuals for NIRC2 just exterior to LkCa $15 \mathrm{c}$ 's predicted position correspond to the forward-scattering peak of the outer disk, not the inner disk. Modified models may better match the combined LkCa 15 data: e.g., faint negative (positive) residuals on the east (west) for the inner outer disk may be eliminated by introducing pericenter offsets (Thalmann et al 2016).
} 
Table 1

Disk Model Parameters

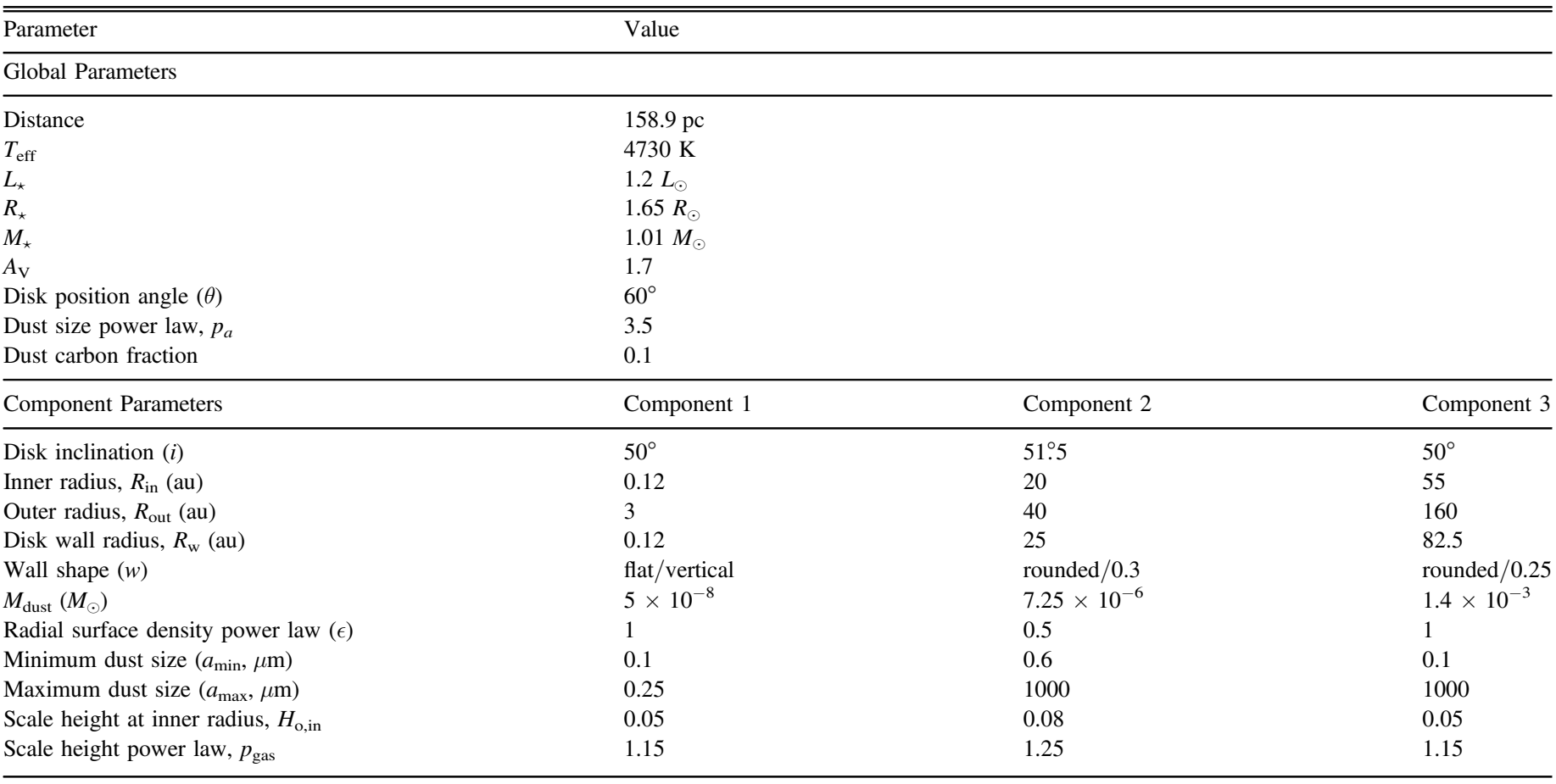

Note. The disk component surface density follows $\Sigma\left(R<R_{\mathrm{w}}\right) \propto R^{-\epsilon} \times \exp \left(-\left(\frac{1-R / R_{\exp }}{w}\right)^{3}\right)$ and $\Sigma\left(R \geqslant R_{\mathrm{w}}\right) \propto R^{-\epsilon}$. The wall shape parameter defines the spatial scale over which the disk surface density increases from $R_{\text {in }}$ to $R_{\mathrm{w}}$. See Mulders et al. (2010, 2013) and Thalmann et al. (2014) for detailed explanations of MCMax3D terminology.

forward-scattering peak. While unresolved, the sub-au disk component requires submicron-sized grains to reproduce the $10 \mu \mathrm{m}$ silicate feature (see also Espaillat et al. 2007). A future paper will thoroughly analyze LkCa 15's disk structures and derive best-fit parameters.

Our modeling also (a) implies that LkCa 15's disk structures should be detectable in optical total intensity imaging and (b) is consistent with the millimeter detection of the outer disk and nondetection of the inner disk. At $0.65 \mu \mathrm{m}$, the inner disk's continuum signal compared to the star (convolved with a Gaussian and integrated within 1.5-2 FWHM) near the reported LkCa $15 \mathrm{~b}$ position in $\mathrm{H}_{\alpha}$ is just slightly lower than $\mathrm{LkCa} 15$ b's reported $\mathrm{H}_{\alpha}$ contrast $\left(\Delta F \sim(2.5-5) \times 10^{-3}\right)$, as is the forward-scattering peak of the outer disk $(\rho \sim 0$ ". 2$)$. The predicted signal of the forwardscattering peak of the inner disk $\left(\rho \sim 00^{\prime \prime} 08\right)$ is comparable in contrast to $\mathrm{LkCa} 15 \mathrm{~b}\left((5-8.5) \times 10^{-3}\right)$. At $7 \mathrm{~mm}$, the model reproduces the outer disk edge's typical intensity, with a characteristic brightness of $\sim 24 \mu \mathrm{Jy}$ beam $^{-1}$ for a beam size of 0 ". 15 ; for a 0. " 07 beam, it accurately predicts that the inner disk $\left(0.5 \mu \mathrm{Jy}\right.$ beam $\left.^{-1}\right)$ would be undetected given a $1 \sigma$ noise floor of $3.6 \mu \mathrm{Jy}_{\text {beam }^{-1}}$.

\section{Discussion}

Instead of protoplanets, our direct images of $\mathrm{LkCa} 15$ obtained with SCExAO/CHARIS show extended, unresolved inner disk emission. Forward-modeling shows that the SCExAO data were capable of distinguishing between disk emission point sources with $K$ band photometry and astrometry reported for LkCa 15's planets by Sallum et al. (2015b). While Kraus \& Ireland (2012) identify concentrated emission sources in SAM data, they use a binary ( $\mathrm{LkCa} 15 \mathrm{~A}+$ companions) light distribution model for image reconstruction, which is valid only if the brightness distribution resembles point sources. Our data show that it does not.

On the other hand, the inner disk signal is comparable to the total flux density reported for $\mathrm{LkCa} 15$ bcd from Sallum et al. (2015b) at $K$ and $L_{\mathrm{p}}$. Thus, we emphasize that the Sallum et al. (2015b) SAM data likely detected the inner disk at multiple wavelengths. Furthermore, the gaps and misalignments between LkCa 15's resolved disk structures, as well as a warp inferred from the sub-au component (Alencar et al. 2018), may be evidence for unseen Jovian planets (Dong \& Fung 2017), which could be detected with future facilities (e.g., the Thirty Meter Telescope; Skidmore et al. 2015).

Our Keck/NIRC2 $L_{\mathrm{p}}$ data obtained between 2009 and 2017 reveal this emission to be static. Based on SAM data taken over a shorter timescale, Sallum et al. (2015b, 2016) argued that LkCa 15 bcd astrometry reveals evidence for orbital motion, although different components are detected in different epochs and the combined astrometry appears consistent with stationary sources given large error bars. While the evaluation of our data is straightforward, several factors may complicate this aspect of SAM data interpretation for $\mathrm{LkCa} 15$. For example, variable $u-v$ coverage between epochs can induce apparent astrometric offsets when a binary model is assumed in the image reconstruction process (C. Caceres 2019, in preparation). Instead of bare stellar photospheres, the calibrators used for $\mathrm{LkCa} 15$ in Sallum et al. (2015b) and especially Kraus \& Ireland (2012) include multiple stars with bright resolved disk emission on the same spatial scale as LkCa 15's disk: some are also highly variable (e.g., GM Aur, UX Tau; Tanii et al. 2012; Oh et al. 2016a). 

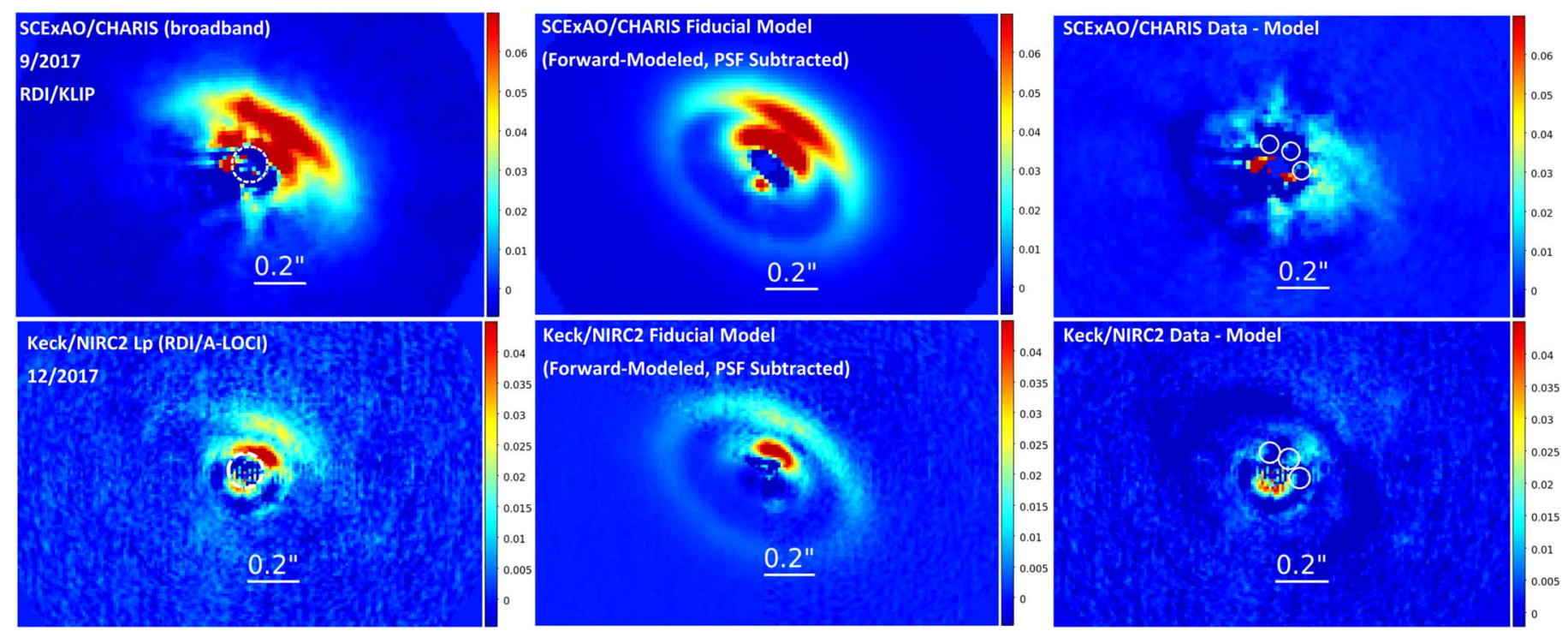

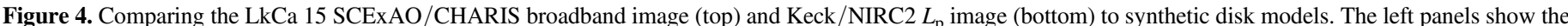

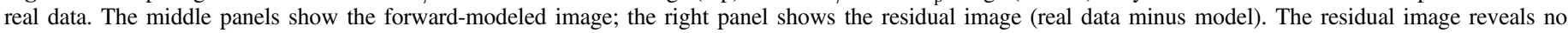
evidence for embedded planets at LkCa 15 bcd's locations (circles). The model is produced as is, not re-scaled in flux to minimize residuals in any data set.

Another common argument is that $\mathrm{LkCa} 15 \mathrm{bcd}$ are too red to be consistent with scattered-light disk emission (Kraus \& Ireland 2012; Ireland \& Kraus 2014). However, for a system with a pre-transitional disk structure like LkCa 15, (a) scattering can be extremely red because (b) the sub-au dust component contributes significantly to the NIR broadband flux and intercepts (and then re-emits) a significant fraction of the starlight (Mulders et al. 2013; Currie et al. 2017b). The light that $\mathrm{LkCa} 15$ 's 20 au scale disk "sees" is then far redder than the star. Indeed, our fiducial disk model successfully reproduces the brightness of the inner dust disk at $K$ and $L_{\mathrm{p}}$. While Sallum et al. (2015b) argued that a disk cannot explain LkCa 15 bc(d) in current SAM data, they use a very simple inclined disk model, not a radiative transfer model. Additionally, from inspection of their Figure 8, the inner component of this model appears to have semimajor and semiminor axes of $\sim 0$ ". 08 and $\sim 0$ ". 05 , which are inconsistent with the larger, spatially resolved, and extended disk as resolved at $K$ and $L_{\mathrm{p}}$ in this study $(0$ ". 2 and 0 "! 1$)$.

Our analyses do not directly refute the claimed single-epoch MagAO $\mathrm{H}_{\alpha}$ detection for $\mathrm{LkCa} 15 \mathrm{~b}$, which technically remains a candidate companion. However, they help strengthen arguments voicing strong skepticism. As LkCa $15 \mathrm{~A}$ itself is bright in $\mathrm{H}_{\alpha}$ due to accretion its disk structures should have an elevated $\mathrm{H}_{\alpha}$ luminosity. Mendigutia et al. (2018) recently found that $\mathrm{LkCa} 15$ 's spectroastrometric signature at $\mathrm{H}_{\alpha}$ is inconsistent with that of a planet but consistent with a disk. They rule out $\mathrm{H}_{\alpha}$ emission from a $\mathrm{LkCa} 15 \mathrm{~b}$ unless the candidate has an $\mathrm{H}_{\alpha}$ contrast fainter than 5.5 mag or a continuum contrast brighter than 6 mag: the $\mathrm{H}_{\alpha}$ photometry and continuum upper limits from Sallum et al. (2015b) are just barely consistent with these spectroastrometric limits. Their predicted emitting region for $\mathrm{H}_{\alpha}$ is $\rho \sim 0$ ". $07-0$ ". 16 , consistent with our resolved images of LkCa 15's inner disk.

Furthermore, SPHERE/ZIMPOL data (Thalmann et al. 2015) and our modeling show that both the inner disk and outer disks are bright, modest-contrast structures and should be detectable at optical wavelengths covering the MagAO $\mathrm{H}_{\alpha}$ observations. Yet Sallum et al. (2015b) did not report a disk detection with MagAO, implying that their $\mathrm{H}_{\alpha}$ planet detection may instead be spurious or a misidentified, partially subtracted piece of the $\mathrm{H}_{\alpha}$-bright disk. Their quoted position for LkCa 15 $\mathrm{b}$ in $\mathrm{H}_{\alpha}$ is conspicuously close to the inner disk's major axis. Given the MagAO observations' poor field rotation $(1.5 \lambda / D$ at 0 ." 1$)$ and negligibly small rotation gap $\left(5^{\circ}\right.$ or $\sim 0.12 \lambda / D$ at 0 ". 1 ), any inclined disk at a comparable separation will suffer severe self-subtraction: its residual emission near the major axis would be preferentially preserved and appear point-like.

Forward-modeling of both a planet and a disk through the MagAO data-as performed to assess HD 100546c (Currie et al. 2015)—could determine which signal better reproduces the images. However, this test is absent from the Sallum et al. (2015b) analysis. The MagAO $\mathrm{H}_{\alpha}$ data are proprietary, not public, preventing any independent verification that the planet hypothesis is preferred. The public availability of archival Keck/NIRC2 data presented here was crucial in assessing evidence for planets orbiting $\mathrm{LkCa} 15$ from aperture masking.

In summary, we rule out the proposed $\mathrm{LkCa} 15$ bcd protoplanets as being primarily responsible for emission seen at small angles in SAM data because the emission (a) would be resolved as separate point sources in the SCExAO data (when it is not) and (b) would rotate between 2009 and 2017 Keck/ NIRC2 data due to the planets' orbital motion (which it does not). Our results also strengthen the argument from Mendigutia et al. (2018) that $\mathrm{H}_{\alpha}$ data also likely identifies a disk, not $\mathrm{LkCa}$ $15 \mathrm{~b}$.

Thus, there is currently no clear, direct evidence for multiple protoplanets orbiting $\mathrm{LkCa} 15$. While the system shows indirect evidence for at least one unseen Jovian planet, the bright inner dust disk impedes the detection of this companion (s). To confirm Jovian companions around $\mathrm{LkCa} 15$ from future observations, the inner disk should be resolved and its effect modeled, removed, and shown to be distinguishable from planets. Protoplanet candidates identified from similar systems should likewise be clearly distinguished from disk emission through multi-wavelength and/or multi-epoch modeling (e.g., Keppler et al. 2018). 
Distinguishing between disk emission and bona fide protoplanets will continue to be a key challenge for the field of direct imaging (e.g., Cieza et al. 2013; Kraus et al. 2013; Sallum et al. 2015a; Ligi et al. 2018; Christiaens et al. 2019; Rich et al. 2019; this work).

We thank Michiel Min for graciously sharing the MCMax3D code and the Subaru and NASA/Keck Time Allocation Committees for their generous support. We thank the anonymous referee for a careful, thoughtful review. Laurent Pueyo, Jun Hashimoto, Christian Thalmann, Catherine Espaillat, Nienke van der Marel, Hannah Jang-Condell, Geoff Bower, and Scott Kenyon provided helpful comments and/or additional, independent assessments of this manuscript. We emphasize the pivotal cultural role and reverence that the summit of Maunakea has always had within the Hawaiian community. We are most fortunate to conduct scientific observations from this mountain. T.C. was supported by a NASA Senior Postdoctoral Fellowship and NASA/Keck grant LK-2663-948181; L.C. was supported by CONICYT-FONDECYT grant No. 1171246. C.C. acknowledges support from project CONICYT PAI/Concurso Nacional Insercion en la Academia, convocatoria 2015, folio 79150049. M.T. is supported by JSPS KAKENHI grant Nos. $18 \mathrm{H} 05442$ and 15H02063. This work utilized the Keck Observatory Archive (KOA), which is operated by the W. M. Keck Observatory and the NASA Exoplanet Science Institute (NExScI), under contract with the National Aeronautics and Space Administration.

\section{ORCID iDs}

Thayne Currie (iD https://orcid.org/0000-0002-7405-3119 Christian Marois (i) https://orcid.org/0000-0002-4164-4182 Lucas Cieza (1D https://orcid.org/0000-0002-2828-1153 Gijs D. Mulders (1) https://orcid.org/0000-0002-1078-9493 Claudio Caceres (1) https://orcid.org/0000-0002-6617-3823 John Wisniewski (iD https://orcid.org/0000-0001-9209-1808 Olivier Guyon (iD https://orcid.org/0000-0002-1097-9908 Timothy D. Brandt (i) https://orcid.org/0000-0003-2630-8073 Jeffrey Chilcote (10) https://orcid.org/0000-0001-6305-7272 Klaus Hodapp (iD https://orcid.org/0000-0003-0786-2140 Frantz Martinache (iD https://orcid.org/0000-0003-1180-4138 Wladimir Lyra (10) https://orcid.org/0000-0002-3768-7542 Motohide Tamura (i) https://orcid.org/0000-0002-6510-0681 Ruben Asensio-Torres (i) https://orcid.org/0000-00032990-0726

Ruobing Dong (ib https://orcid.org/0000-0001-9290-7846 Misato Fukagawa (i) https://orcid.org/0000-0003-3500-2455 Tomoyuki Kudo (iD https://orcid.org/0000-0002-9294-1793 Jungmi Kwon (ib https://orcid.org/0000-0003-2815-7774 Taichi Uyama (iD https://orcid.org/0000-0002-6879-3030

\section{References}

Alencar, S. H. P., Bouvier, J., Donati, J.-F., et al. 2018, A\&A, 620, 195 Andrews, S., Rosenfeld, K., Wilner, D. J., \& Bremer, M. 2011, ApJL, 742, L5
Brandt, T. D., Rizzo, M., Groff, T., et al. 2017, JATIS, 3, 048002 Christiaens, V., Casassus, S., Absil, O., et al. 2019, MNRAS, in press (arXiv:1905.01860)

Cieza, L., Lacour, S., Schreiber, M., et al. 2013, ApJL, 762, L12

Currie, T., Brandt, T. D., Uyama, T., et al. 2018a, AJ, 156, 291 Currie, T., Brittain, S., Grady, C., et al. 2017a, RNAAS, 1, 40

Currie, T., Burrows, A., Girard, J., et al. 2014a, ApJ, 795, 133

Currie, T., Burrows, A., Itoh, Y., et al. 2011, ApJ, 729, 128

Currie, T., Cloutier, R., Brittain, S., et al. 2015, ApJL, 814, L27

Currie, T., Daemgen, S., Debes, J., et al. 2014b, ApJL, 780, L30

Currie, T., Debes, J., Rodigas, T., et al. 2012, ApJL, 760, L32

Currie, T., Guyon, O., Tamura, M., et al. 2017b, ApJL, 836, L15

Currie, T., Kasdin, N. J., Groff, T., et al. 2018b, PASP, 130, 044505

Dodson-Robinson, S., \& Salyk, C. 2011, ApJ, 738, 131

Dong, R., \& Fung, J. 2017, ApJ, 835, 146

Espaillat, C., Calvet, N., D’Alessio, P., et al. 2007, ApJL, 670, L135

Espaillat, C., Furlan, E., D’Alessio, P., et al. 2011, ApJ, 728, 49

Furlan, E., Forrest, W. J., Sargent, B. A., et al. 2009, ApJ, 706, 1194

Groff, T. D., Chilcote, J., Brandt, T. D., et al. 2017, Proc. SPIE, 10400, 1040016

Groff, T. D., Kasdin, N. J., Limbach, M., et al. 2015, Proc. SPIE, 9605, $96051 \mathrm{C}$

Ireland, M., \& Kraus, A. 2014, in IAU Symp. 299, Exploring the Formation and Evolution of Planetary Systems, ed. M. Booth, B. C. Matthews, \& J. R. Graham (Cambridge: Cambridge Univ. Press), 199

Isella, A., Chandler, C. J., Carpenter, J. M., Perez, L. M., \& Ricci, L. 2014, ApJ, 788, 129

Jovanovic, N., Martinache, F., Guyon, O., et al. 2015, PASP, 127, 890

Kenyon, S. J., Gomez, M., \& Whitney, B. 2008, Handbook of Star-forming Regions

Keppler, M., Benisty, M., Muller, A., et al. 2018, A\&A, 617, 44

Kraus, A., \& Ireland, M. 2012, ApJ, 745, 5

Kraus, A., Ireland, M., Martinache, F., \& Hillenbrandt, L. 2011, ApJ, 731, 8

Kraus, S., Ireland, M., Sitko, M., et al. 2013, ApJ, 768, 80

Lacour, S., Tuthill, P., Amico, P., et al. 2011, A\&A, 532, L72

Lafreniére, D., Marois, C., Duyon, R., et al. 2007, ApJ, 660, 770

Ligi, R., Vigan, A., Gratton, R., et al. 2018, MNRAS, 437, 1774

Marois, C., Correia, C., Galicher, R., et al. 2014, Proc. SPIE, 9148, 91480

Marois, C., Lafreniére, D., Duyon, R., et al. 2006, ApJ, 641, 556

Marois, C., Lafreniére, D., Macintosh, B., \& Doyon, R. 2008a, ApJ, 673, 647

Marois, C., Macintosh, B., Barman, T., et al. 2008b, Sci, 322, 1348

Marois, C., Macintosh, B., \& Veran, J.-P. 2010a, Proc. SPIE, 7736, 77361J

Marois, C., Zuckerman, B., Konopacky, Q., et al. 2010b, Natur, 468, 1080

Mawet, D., Milli, J., Wahhaj, Z., et al. 2014, ApJ, 792, 97

Mendigutia, I., Oudmaijer, R. D., Schneider, P. C., et al. 2018, A\&A, 618, L9

Min, M., Dullemond, C. P., Dominik, C., de Koter, A., \& Hovenier, J. W. 2009, A\&A, 497, 155

Mulders, G., Dominik, C., \& Min, M. 2010, A\&A, 512, 11

Mulders, G., Paardekooper, S.-J., Panic, O., et al. 2013, A\&A, 557, 68

Oh, D., Hashimoto, J., Carson, J. C., et al. 2016a, ApJL, 831, L7

Oh, D., Hashimoto, J., Tamura, M., et al. 2016b, PASJ, 68, L3

Pueyo, L. 2016, ApJ, 824, 117

Ribas, I., Espaillat, C., Macias, E., et al. 2017, ApJ, 849, 63

Rich, E., Wisniewski, J., Currie, T., et al. 2019, ApJ, 875, 38

Rodriguez, J., Andsell, M., Oelkers, R., et al. 2017, ApJ, 848, 97

Sallum, S., Eisner, J., Close, L., et al. 2016, Proc. SPIE, 9907, 99070D

Sallum, S., Eisner, J., Close, L. M., et al. 2015a, ApJ, 801, 85

Sallum, S., Follette, K., Eisner, J., et al. 2015b, Natur, 527, 342

Skidmore, W., TMT International Science Development Teams, Science Advisory Committee, TMT, et al. 2015, RAA, 15, 1945

Soummer, R., Pueyo, L., \& Larkin, J. 2012, ApJL, 755, L28

Tanii, R., Itoh, Y., Kudo, T., et al. 2012, PASJ, 64, 124

Thalmann, C., Grady, C., Goto, M., et al. 2010, ApJL, 718, L87

Thalmann, C., Janson, M., Garufi, A., et al. 2016, ApJL, 828, L17

Thalmann, C., Mulders, G. D., Hodapp, K., et al. 2014, A\&A, 566, 5

Thalmann, C., Mulders, G. D., Janson, M., et al. 2015, A\&A, 808, L41

Tuthill, P., Lloyd, J., Ireland, M., et al. 2006, Proc. SPIE, 6272, 62723A 